\title{
CSF xanthochromia analysis takes longer following centralisation of the laboratories testing samples - how can we improve the time to result?
}

\author{
Sean Martin, Melanie Page, lan Godber, Calum McGregor \\ Wishaw General Hospital, Wishaw, Lanarkshire, United Kingdom
}

\begin{abstract}
Lumbar puncture is an essential tool for excluding subarachnoid haemorrhage. In August 2012, the laboratory at which cerebrospinal fluid (CSF) is analysed for xanthochromia in Lanarkshire was centralised at Hairmyres (East Kilbride, UK). Prior to this, each of the three hospitals analysed their own specimens.
\end{abstract}

We aim to assess whether or not the change in xanthochromia processing has resulted in diagnostic delay at Wishaw General Hospital in the assessment of CT negative possible subarachnoid haemorrhage. We subsequently assessed the impact of a strategy to minimise any delay, i.e. increasing laboratory processing hours.

Patients undergoing CSF analysis for xanthochromia were identified directly from the laboratory database. Time of lumbar puncture, and time of xanthochromia results were obtained from the hospital's laboratory computer system. Data were analysed using a commercially available statistical software programme (Microsoft Excel). Audit was repeated after the change to a centralised laboratory, and again following the increased laboratory working hours.

Mean time from lumbar puncture to availability of xanthochromia result was significantly longer following the laboratory change ( $20.8 \pm 3.5$ hours post [ $n=35$ ] vs. $12.5 \pm 3.0$ pre, $p=0.01$ [ $n=17]$ ). However, following a change in the laboratory's practice, there was no improvement $(19.8 \pm 3.4$ hours post practice change $[n=35])$, and this remained significantly longer when compared to the original laboratory set-up $(p=0.025)$.

The change in laboratory processing CSF samples for xanthochromia in Lanarkshire resulted in a significant delay in analysing the samples. Attempts by the laboratory to extend processing hours did not make any significant improvement, but having expanded our knowledge of the issues, further measures are now planned to minimise delays in the future.

Centralisation of laboratory services for CSF analysis, whilst cheaper, may impact negatively on clinical care.

\section{Problem}

Prior to August 2012, the laboratory at Wishaw General Hospital processed all of the cerebrospinal fluid (CSF) samples sent for xanthochromia on-site. This service was subsequently centralised for the whole health board at another hospital - requiring samples once taken, to be sent by taxi to another site. Following this laboratory change, clinicians suspected that results were taking longer to become available.

This was compounded by the fact that samples are not processed out of hours, due to staff levels in the laboratories overnight. There is one on-call person in the laboratory, whose function is to analyse urgent specimens. CSF xanthochromia analysis is a labour intensive process that requires complete concentration, and attention from start to finish. Starting to process a xanthochromia scan in the "on-call" period might lead to the member of staff being disturbed to answer phone calls or other such interruptions with the attendant danger of loss of concentration when analysing important samples, and may also lead to a delay in processing other emergency samples.
We also posit that lumbar punctures are often "put off" by doctors until the end of the day as not being urgent and time consuming, not least because our receiving unit is better staffed in the afternoon. This means that samples do not reach the laboratory in working hours and are consequently often not analysed the same day. Indeed, the late afternoon period is the busiest in the laboratory - working flat out processing hospital and GP blood samples, which may also delay CSF processing.

Another possible compounding factor is that CT scans are often not requested until the morning ward round, meaning that the time taken for the radiology department to carry out and report CT brain scans delays the indication for a lumbar puncture.

\section{Background}

Lumbar puncture is an essential tool for excluding subarachnoid haemorrhage in those patients with a suggestive history and a negative CT scan - especially in late presentations (1). On this basis, it is a very important diagnosis to make or refute. 
Initially, all CSF samples sent for xanthochromia analysis in Lanarkshire were analysed on-site at the laboratory in which the sample was obtained.

Following centralisation of this service, samples from the entire health board were sent to a single laboratory, and samples received at or before $4 \mathrm{pm}$ were analysed that day.

Recognising that the time-frame for analysis was negatively affected by this change in laboratory structure, we were able to extend the period of time for analysis to $6 \mathrm{pm}$, in an attempt to analyse more of the samples obtained and received by the laboratory in the afternoon and evening.

\section{Baseline Measurement}

Patients that had CSF samples analysed for xanthochromia prior to laboratory change were identified directly from laboratory records. Time of lumbar puncture, and time of result availability were obtained from the hospital computer system. The average sample turn around time was $12.5 \pm 3.0$ hours.

\section{Design}

The intervention here was a laboratory change, and the analysis of delay in diagnosis was made retrospectively in order to quantify the impact that it may have had on clinical work.

Our main study outcome was a simple one to collect data for, so our preliminary results were available very quickly. It was clear that the laboratory change had resulted in a lengthy increase in time from lumbar puncture to xanthochromia result. This was immediately discussed with the Lead Biochemist for the health board with a view to looking at ways to reduce this time.

The obvious answer was to introduce out-of-hours xanthochromia analysis. However, this would be an expensive endeavour since the staffing levels in the biochemistry laboratory preclude this currently, and so, an extra trained member of staff would have to be paid for this to occur. Another option discussed was sending the out-ofhours samples by taxi to another health board for analysis - again a very costly endeavour.

We settled on extending the hours of analysis for receipt of samples up until $6 \mathrm{pm}$, whereas previously it had been $4 \mathrm{pm}$, in order to be able to analyse samples received by the larboratory later in the afternoon. Unfortunately, this did have made any effect on the time taken for results to be made available.

We then looked at the time samples were taken and sent to the laboratories. Realising that this was largely later afternoon (which do not reach the lab until after hours), we have been able to recommend that lumbar punctures are prioritised for the morning

Data were then analysed using a commercially available statistical software programme (Microsoft Excel). Results are expressed as proportions $(\%)$, mean values \pm SEM, or median as appropriate.
Student's t-test was used to compare numerical variables between cohorts. $P$ values of $<0.025$ were considered to be statistically significant.

\section{Strategy}

As soon as we obtained preliminary results that showed a highly significant increase in time to result following the new system, we discussed the issue with the lead consultant in biochemistry for the health board. This resulted in samples being received by the laboratory later in the evening, in an effort to provide a comparable service to that when samples were analysed on-site. We also tried to improve communication between clinicians and laboratory staff. However, this did not show any real improvement.

This prompted us to look at other options: we stratified CSF collection time and the respective time taken to get results for these samples. This showed us that lumbar punctures undertaken earlier in the day were reported much more quickly than those done in the late afternoon - but it also showed us that the majority of lumbar punctures were carried out in the late afternoon.

On this basis, we have recommended that lumbar punctures to exclude subarachnoid haemorrhage should be prioritised following ward rounds so that results are available quickly. This is important for two reasons: in those few positive cases, the patients can be referred to neurosurgery timeously, and secondly, those patients with negative results can be reassured and discharged without the requirement for an overnight hospital stay, simply awaiting a single test result.

In order to disseminate this information to the relevant staff, we are in the process of introducing a "Lumbar Puncture Pack" for all junior medical staff. This includes: a clinical skills teaching session, written document, video, and models for practise. Furthermore, to ensure that this is sustainable, we intend to audit the performance of lumbar puncture before and after this intervention.

\section{Results}

Pre laboratory change (a): mean time to result from collection, $12.46 \pm 3.02$ hours

Post laboratory change (b): mean time to result from collection, $20.76 \pm 3.52$ hours

Post laboratory change in practice (c): mean time to result from collection, $19.84 \pm 3.36$ hours

Comparing these:

a vs. $b: p=0.011$

a vs. c: $p=0.025$

b vs. $c: p=0.74$ 


\section{BMJ Quality Improvement Reports}

a vs. $b+c: p=0.005$

These data clearly show that the change in laboratory practise resulted in an increased length of time between taking a CSF sample and receiving the xanthochromia result. This effect was not abrogated by the changes we attempted to make in processing hours at the new laboratory - and indeed was still significantly slower than the original configuration.

Subgroup analysis of the collated post-lab change groups $(n=70)$ shows that the largest proportion (49\%; Figure, blue line) of lumbar punctures are undertaken in the afternoon (between 3pm and 6pm), and that samples taken during this time period take the longest to give a result, $22.5 \pm 1.8$ hours. By comparison, those samples taken earlier (between 9am and 3pm) have conclusive results much more quickly (13.2 \pm 2.9 hours $p=0.004$; Figure, red line), though are carried out in this time period more infrequently $(20 \%)$.

Furthermore, samples taken early in the day (0900-1500), when compared with those taken out-of-hours (1800-0900) obtain results significantly quicker, $p=0.022$. Indeed, samples taken in this time period achieve results more quickly than at all other time points combined, $p=0.004$.

See supplementary file: ds2143.png - "LP and Results By Time of Day"

\section{Lessons and Limitations}

We learned several lessons while undertaking this piece of work:

1. Centralisation of laboratory services, whilst cheaper, may impact negatively on clinical care.

2. Lumbar punctures are most often performed between 3pm and $6 \mathrm{pm}$ at our centre.

3. Lumbar punctures may "put off" by doctors until the afternoon when their jobs list is less busy, and this could be a possible explanation for lumbar punctures being carried out in the afternoon, as we observed.

4. CT scans must be done before a lumbar puncture is carried out - this may push the time back until later in the day.

We feel that our intervention has the potential to save a large amount of money (though we have not been able to quantify this at present) for the trust since there appear to be lots of patients that unnecessarily have their discharge delayed awaiting a xanthochromia result. If we are able, by implementing free, simple measures to speed up this result from a "next-day" to a largely "same-day" service, the amount of money saved could be large given the high cost of hospital beds.

\section{Conclusion}

Indeed, the change in laboratory processing CSF samples for xanthochromia in Lanarkshire has resulted in a significant delay in a diagnosis of a potentially life-threatening presentation. Attempts by the laboratory to extend processing hours have not made any significant improvement in turning around these samples.

We have shown that lumbar punctures performed earlier in the day are analysed quicker, therefore we are planning to implement strategies to ensure that lumbar punctures are performed earlier: by prioritising these tests in the receiving unit, and by better education of junior staff.

In a time where the NHS is centralising services for cost efficiency without change in care, it is important to consider that the delays which may be lent by this incur the additional costs of delayed discharges and, significantly, delayed diagnoses. However, it should be noted that cost efficiency is not the only concern in centralising services: it also concentrates staff expertise, allowing development and maintenance of skills in a complex area, so offering more than monetary benefit.

\section{References}

1. Hutchinson PJ, Kirkpatrick PJ. Headache: Diagnosing subarachnoid hemorrhage: are CT scans enough? Nature Reviews Neurology. 2012;(8):126-127

\section{Declaration of interests}

Nothing to declare.

\section{Acknowledgements}

Dr Kieran McKenna, Consultant Physician 